OPEN

SUBJECT AREAS:

CHEMICAL PHYSICS

CONDENSED-MATTER PHYSICS

Received

7 November 2014

Accepted

9 December 2014

Published

12 January 2015

Correspondence and requests for materials should be addressed to

J.-T.W. (wjł@aphy. iphy.ac.cn)

\section{Crystalline structures of polymeric hydrocarbon with 3,4-fold helical chains}

\author{
Chao-Sheng Lian', Han-Dong Li \& Jian-Tao Wang'
}

'Beijing National Laboratory for Condensed Matter Physics, Institute of Physics, Chinese Academy of Sciences, Beijing 100190, China, ${ }^{2}$ State Key Laboratory of Environmental Criteria and Risk Assessment, Chinese Research Academy of Environmental Sciences, Beijing 100012, China.

Molecular hydrocarbons are well-known to polymerize under pressure to form covalently bonded frameworks. Here we predict by $a b$ initio calculations two distinct three-dimensional hydrocarbon crystalline structures composed of 3 -fold and 4 -fold helical $\mathrm{CH}$ chains in rhombohedral $(R \overline{\mathbf{3}})$ and tetragonal $\left(I 4_{1} / a\right)$ symmetry, respectively. Both structures with $1: 1$ stoichiometry are found to be energetically more favorable than solid acetylene and cubane, and even more stable than benzene II solid at high pressure. The calculations on vibrational, electronic, and optical properties reveal that the new chiral hydrocarbons are dynamically stable with large bulk moduli around $200 \mathrm{GPa}$, and exhibit a transparent insulating behavior with indirect band gaps of $5.9 \sim 6.7 \mathrm{eV}$ and anisotropic adsorption spectra. Such forms of hydrocarbon, once synthesized, would have wide applications in mechanical, optoelectronic, and biological materials.

T he exploration of new technological materials is an enduring topic in high-pressure physics and chemistry ${ }^{1}$ and simple molecular solids are rising as potential precursors due to their high compressibility ${ }^{2}$. It has been shown that many unsaturated hydrocarbons form molecular crystals that polymerize under pressure into extended covalently bonded structures ${ }^{3-10}$, which can generally be recovered to ambient conditions unlike the cases in $\mathrm{N}_{2}, \mathrm{CO}_{2}$, and $\mathrm{N}_{2} \mathrm{O}$ where polymeric phases decompose at ambient pressure ${ }^{11-13}$. Ethylene ${ }^{3,4}$ and acetylene ${ }^{5,6}$ transform to linear polymers at 1-6 GPa through the opening of double and triple bonds. With the aromatic character lost, benzene polymerizes around $23 \mathrm{GPa}$ in the amorphization form at room temperature ${ }^{7-9}$. A friable and colorless compound has been obtained by heating benzene above $680 \mathrm{~K}$ between 7 and $12 \mathrm{GPa}^{10}$, while its structure is unknown so far. It is confirmed in high-pressure studies of both acetylene ${ }^{6}$ and benzene ${ }^{9}$ that laser irradiation will promote the generation of saturated network polymers with a composition close to $(\mathrm{CH})_{n}$.

Nowadays, there is an increasing theoretical attention paid to the nonmolecular hydrocarbon crystalline phases. In addition to the well-known one-dimensional (1D) polyethylene and polyacetylene, many isomeric $2 \mathrm{D}$ covalently bonded hydrocarbons called graphane were suggested in the last decade ${ }^{14-16}$. Wen et al. ${ }^{17}$ found from ab initio calculations that benzene phases under pressure transform to ordered $\mathrm{CH}$ structures consisting of $1 \mathrm{D}$ or $2 \mathrm{D}$ arrays of $\mathrm{C}_{6} \mathrm{H}_{6}$ rings bridged by $s p^{3} \mathrm{C}-\mathrm{C}$ bonds. This has greatly stimulated the exploration of other possible structures of polymeric hydrocarbon, which could be synthesized by applying high pressure or temperature. Using evolutionary algorithm structure searches, several 3D graphane $\mathrm{CH}$ structures with different graphane stackings had been reported ${ }^{18}$. In a recent study ${ }^{19}$, we predicted a diamond-like 3D cubic hydrocarbon crystal, $K_{4}-\mathrm{CH}$, through hydrogenating the hypothetical $K_{4}$-carbon ${ }^{20}$. Subsequently, a saturated hexagonal phase of Hex-CH was also proposed from the compressed benzene ${ }^{21}$. It has been established that for the $\mathrm{CH}$ system with $1: 1$ stoichiometry, the fully saturated polymeric $\mathrm{CH}$ phases are thermodynamically more stable than any phase retaining discrete molecules ${ }^{17,18}$.

In this paper, we report by ab initio calculations two novel 3D hydrocarbon framework structures that comprise 3 -fold and 4 -fold helical $\mathrm{CH}$ chains in rhombohedral $(R \overline{3})$ and tetragonal $\left(I 4_{1} / a\right)$ symmetry, respectively, which are derived from the fully hydrogenation of our recently found all-s $s p^{2}$ bonded chiral carbene ${ }^{22}$. The structure stability, electronic and optical properties are examined up to $50 \mathrm{GPa}$. Both chiral crystalline phases are confirmed to be energetically more favorable than solid acetylene and cubane, and significantly stabilized with increasing pressure relative to the benzene II solid. The calculated vibrational and mechanical properties reveal their dynamical stability and large bulk modulus of $\sim 200 \mathrm{GPa}$. Electronic and optical calculations show the widegap insulating behavior typical of saturated hydrocarbon and the unique anisotropic adsorption spectra. Our predictions can be expected to greatly stimulate future experiments to synthesize these new phases. 


\section{Results}

Our newly identified chiral crystalline forms of hydrocarbon are depicted in Fig. 1. The 4-fold chiral structure [Fig. 1(a)] is bodycentered tetragonal in $I 4_{1} / a$ symmetry, and the optimized lattice parameters are $a=6.106 \AA$ and $c=4.146 \AA$, with $\mathrm{C}$ and $\mathrm{H}$ atoms occupying 16f $(0.2189,0.1188,0.8213)$ and $16 f(0.0770,0.1018$, 0.6617) Wyckoff positions, respectively. This form has eight $\mathrm{CH}$ units in the primitive cell and we refer to it as the $T_{8}-\mathrm{CH}$. The 3-fold chiral structure in $R \overline{3}$ symmetry, hereafter named as $R_{6}-\mathrm{CH}$, has a rhombohedral lattice and six $\mathrm{CH}$ units per primitive cell. In hexagonal representation [Fig. 1(b)], its equilibrium lattice parameters are estimated to be $a=7.392 \AA$ and $c=3.671 \AA$ with $18 \mathrm{f}(0.4202$, $0.0351,0.0448) \mathrm{C}$ and $18 \mathrm{f}(0.1816,0.5314,0.4693) \mathrm{H}$ positions. As shown in Fig. 1, for both $T_{8}-\mathrm{CH}$ and $\mathrm{R}_{6}-\mathrm{CH}$ structure, the helical $\mathrm{CH}$ chains are formed along the $c$ axis, and each chain is connected to neighboring chains of opposite chirality (left-handed indicated by $\mathrm{S}$ and right-handed $\mathrm{R}$ ) by $\mathrm{C}-\mathrm{C}$ covalent bonds. The intrachain and interchain C-C bond lengths are respectively 1.559 and $1.564 \AA$ in $T_{8}-\mathrm{CH}, 1.561$ and $1.543 \AA$ in $R_{6}-\mathrm{CH}$, which are all close to $1.530 \AA$ in diamond ${ }^{19}$. Hence, the two chiral hydrocarbons here can be considered as diamond-like $\mathrm{CH}$ phases similar to the previously proposed $K_{4}-\mathrm{CH}^{19}$ and $\mathrm{Hex}-\mathrm{CH}^{21}$; they all adopt a fully $3 \mathrm{D}$ framework with saturated nature of $s p^{3}$ carbon.

Figure 2(a) shows the calculated total energy versus volume curves of various hydrocarbon phases with $\mathrm{CH}$ stoichiometry of $1: 1$. We can see that the $T_{8}-\mathrm{CH}$ structure is as stable as $K_{4}-\mathrm{CH}$, with energy about 0.47 and $1.16 \mathrm{eV} / \mathrm{CH}$ lower than solid cubane ${ }^{23,24}$ and acetylene $^{25}$, respectively. Compared with $T_{8}-\mathrm{CH}$, the $R_{6}-\mathrm{CH}$ structure has lower energy (close to Hex-CH), being even more stable than the benzene $\mathrm{II}^{26}$ crystal and its hypothetical layered polymer reported by Wen et al. ${ }^{17}$ It is noticeable that the equilibrium volumes of $K_{4}$ $\mathrm{CH}, \mathrm{Hex}-\mathrm{CH}, \mathrm{T}_{8}-\mathrm{CH}$, and $R_{6}-\mathrm{CH}$ are in the range 9.6-9.9 $\AA^{3} / \mathrm{CH}$, much smaller than $14.1-20.5 \AA^{3} / \mathrm{CH}$ of benzene II and solid cubane and acetylene (see Table I). These results suggest great potential for synthesizing the low-energy $\mathrm{CH}$ structures through compression of metastable molecular phases of hydrocarbon. Furthermore, we find that among all $\mathrm{CH}$ systems considered here the most stable phase is the layered graphane $\mathrm{I}^{18}$ crystal in an AA stacking $(0.04 \mathrm{eV} / \mathrm{CH}$ lower in energy than graphane III). The less favorable energetic state of diamond-like $\mathrm{CH}$ phases compared to layered graphane is likely attributed to the stronger steric interactions of hydrogens ${ }^{18}$, as evidenced by the nearest $\mathrm{H}$-H distances of $1.56 \AA$ for $T_{8}-\mathrm{CH}$ and $1.79 \AA$ for $R_{6}-\mathrm{CH}$ being shorter than $2.51 \AA$ for graphane I.

To understand the pressure effect, the enthalpy difference of each phase to that of benzene II is presented in Fig. 2(b) in a wide pressure range $0-50 \mathrm{GPa}$. It is found consistent with previous calculations ${ }^{18}$ that instead of graphane I, graphane III becomes the most stable phase under pressures above $12 \mathrm{GPa}$. For diamond-like $\mathrm{CH}$ phases, an increasing stabilization with pressure can be seen relative to benzene II and the related polymer. Above 3.5 and 7.0 GPa, the $T_{8}-\mathrm{CH}$ and $K_{4}-\mathrm{CH}$ phases (enthalpically almost degenerate in $0-10 \mathrm{GPa}$ ) become more stable than benzene II and benzene II polymer, respect- (a)

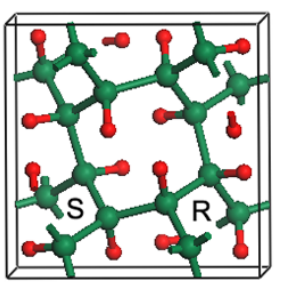

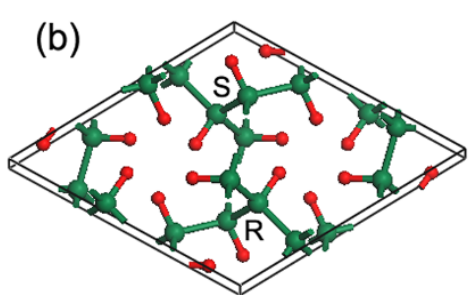

Figure 1 Schematic representation of the chiral crystalline structures of $T_{8}-\mathrm{CH}(\mathrm{a})$ and $\mathrm{R}_{6}-\mathrm{CH}(\mathrm{b})$. Carbon atoms are shown as large olive spheres, and hydrogen atoms are shown as small red spheres. $\mathrm{R}$ and $\mathrm{S}$ refer to right- and left-handed helical CH chains, respectively.
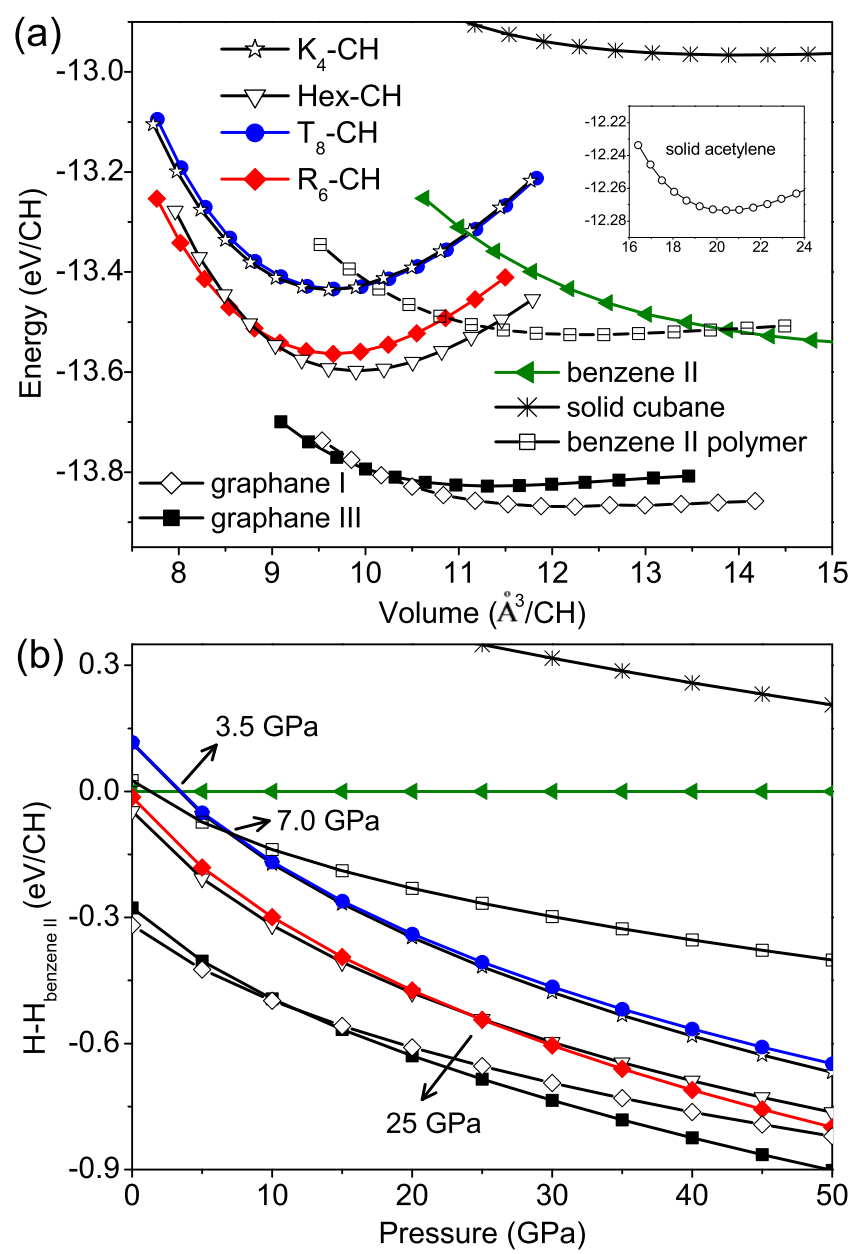

Figure $2 \mid$ (a) Energy versus volume curves for $K_{4}-\mathrm{CH}$, Hex- $\mathrm{CH}, \mathrm{T}_{8}-\mathrm{CH}$, $R_{6}-\mathrm{CH}$, molecular phases of hydrocarbon such as benzene II and solid cubane, benzene II polymer, and graphane I and III. The inset shows the data for solid acetylene. (b) Enthalpy difference with respect to benzene II for the various $\mathrm{CH}$ phases as a function of pressure.

ively. Moreover, the hexagonal phases of $\mathrm{R}_{6}-\mathrm{CH}$ and $\mathrm{Hex}-\mathrm{CH}$ are more favorable than $T_{8}-\mathrm{CH}$ or $K_{4}-\mathrm{CH}$ in enthalpy by about $0.15 \mathrm{eV} /$ $\mathrm{CH}$ in the whole pressure range. Meanwhile, $R_{6}-\mathrm{CH}$ is found to be preferable to Hex-CH above $25 \mathrm{GPa}$, and to compete with graphane I above $50 \mathrm{GPa}$. In view of the above enthalpy results, the experimental syntheses of diamond-like $\mathrm{CH}$ phases are thermodynamically possible.

We have fitted the energy versus volume data to the Murnaghan equation of $\operatorname{state}^{27}$ to obtain the bulk moduli $\left(B_{0}\right)$ of different hydrocarbons, as listed in Table I. The predicted $B_{0}$ of diamond-like $\mathrm{CH}$ phases are significantly higher than those of molecular hydrocarbons, with $T_{8}-\mathrm{CH}$ and $R_{6}-\mathrm{CH}$ having the values of 201.7 and 185.2 GPa, respectively. Note that for benzene II we calculate the bulk modulus to be $B_{0}=9.8 \mathrm{GPa}$, which is close to the reported experimental value of $\sim 5.5 \mathrm{GPa}^{28}$.

Phonon calculations give a criterion for the structure stability of a crystal. Therefore, we calculated phonon dispersion curves for the $T_{8}-\mathrm{CH}$ and $\mathrm{R}_{6}-\mathrm{CH}$ phases at $0 \mathrm{GPa}$, as presented in Fig. 3(a) and 3 (b). The absence of imaginary frequency modes indicates that these two chiral structures are dynamically stable. High frequency $\mathrm{C}-\mathrm{H}$ stretching phonon modes emerge around 3047 and $2992 \mathrm{~cm}^{-1}$ for $T_{8}-\mathrm{CH}$ and $\mathrm{R}_{6}-\mathrm{CH}$, respectively, which can be compared with the observed broad infrared peaks at 2950 and $2920 \mathrm{~cm}^{-1}$ (assigned to the $\mathrm{C}-\mathrm{H}$ stretching modes involving $s p^{3}$ carbon atoms) for amorphous samples recovered from compressed acetylene $e^{6}$ and benzene $e^{8,9}$. 
Table I Calculated equilibrium structural parameters (space group, lattice parameters $a, b$, and $c$, volume $V_{0}$, bond lengths $d_{C-} d$, total energy $E_{\text {tot }}$, bulk modulus $B_{0}$, and electronic band gap $E_{g}$ for various hydrocarbon phases at zero pressure. Energies are given relative to that of graphane I. For benzene II and benzene II polymer, $\beta=110.62$ and $97.49^{\circ}$, respectively. The $\mathrm{C}-\mathrm{H}$ bond lengths are around $1.10 \AA$ for all phases

\begin{tabular}{|c|c|c|c|c|c|c|c|c|}
\hline Structure & Space group & $a, b(\AA)$ & $c(\AA)$ & $\mathrm{V}_{0}\left(\AA^{3} / \mathrm{CH}\right)$ & $d_{C-C}(\AA)$ & $\mathrm{E}_{\text {tot }}(\mathrm{eV} / \mathrm{CH})$ & $B_{0}(G P a)$ & $\mathrm{E}_{g}(\mathrm{eV})$ \\
\hline $\mathrm{K}_{4}-\mathrm{CH}$ & 12,3 & 4.252 & & 9.62 & 1.565 & 0.43 & 198.4 & 6.07 \\
\hline $\mathrm{Hex}-\mathrm{CH}$ & $R \overline{3} m$ & 5.974 & 5.764 & 9.92 & $1.542,1.575$ & 0.27 & 175.2 & 6.54 \\
\hline $\mathrm{T}_{8} \mathrm{CH}$ & $14_{1} / a$ & 6.106 & 4.146 & 9.67 & $1.559,1.564$ & 0.43 & 201.7 & 6.67 \\
\hline$R_{6}-\mathrm{CH}$ & $R \overline{3}$ & 7.392 & 3.671 & 9.66 & $1.543,1.561$ & 0.30 & 185.2 & 5.88 \\
\hline Benzene II & $P 2_{1} / c$ & $5.382,5.338$ & 7.457 & 16.96 & 1.387 & 0.32 & 9.8 & 5.14 \\
\hline Solid cubane & $R \overline{3}$ & 5.918 & 10.991 & 14.07 & 1.557 & 0.90 & 18.9 & 6.60 \\
\hline Solid acetylene & Cmca & $4.978,5.713$ & 5.742 & 20.49 & 1.208 & 1.59 & 9.1 & 5.43 \\
\hline Benzene II polymer & $P 2_{1} / c$ & $4.449,3.713$ & 8.667 & 12.53 & $1.517-1.551$ & 0.34 & 31.3 & 5.05 \\
\hline Graphane III & Cmca & $2.515,9.502$ & 3.745 & 11.51 & $1.524,1.522$ & 0.04 & 30.6 & 4.76 \\
\hline Graphane I & $P \overline{3} m 1$ & 2.506 & 4.503 & 12.41 & 1.515 & 0.00 & 19.7 & 4.87 \\
\hline
\end{tabular}

In addition, we also checked the phonon dispersion for both chiral phases under pressure confirming their dynamical stability up to at least $50 \mathrm{GPa}$.

The electronic band structure calculations within the hybrid functional method $^{36}$ have demonstrated the insulating nature of the two chiral saturated $\mathrm{CH}$ phases. The valence band maximums of $T_{8}-\mathrm{CH}$ [Fig. 3(c)] and $R_{6}$-CH [Fig. 3(d)] are at the $\Gamma$ and A points in the Brillouin zone while the conduction band minimums are along the $\mathrm{Z}-\mathrm{X}$ direction and at the $\mathrm{M}$ point, giving indirect band gaps of 6.67 and $5.88 \mathrm{eV}$ at $0 \mathrm{GPa}$, respectively. Hence, both chiral phases are predicted to be optically transparent as previously proposed $K_{4}$-CH $(6.07 \mathrm{eV})$ and $\mathrm{Hex}-\mathrm{CH}(6.54 \mathrm{eV})$. We have further explored the electronic properties at increasing pressures. The calculated band
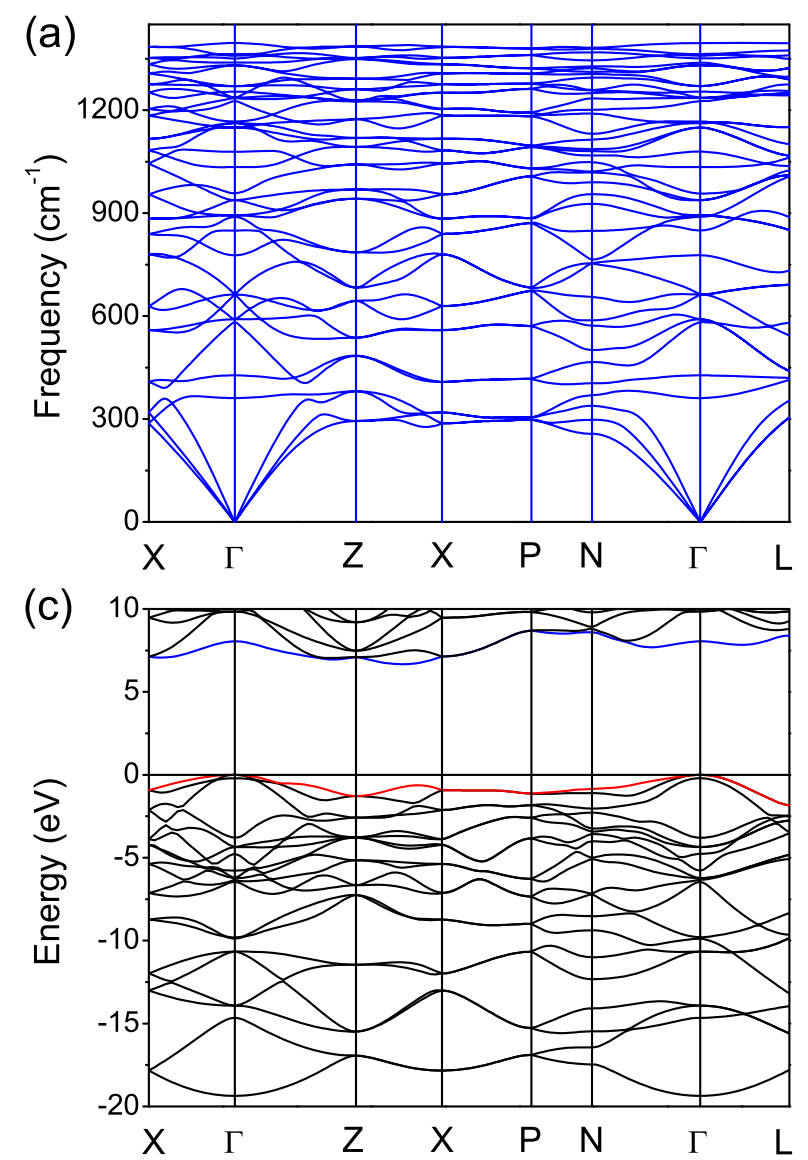

gaps as a function of pressure for $\mathrm{K}_{4}-\mathrm{CH}$, Hex- $\mathrm{CH}, \mathrm{T}_{8}-\mathrm{CH}$, and $R_{6}$ - $\mathrm{CH}$ are shown in Fig. 4(a). According to the results, $T_{8}-\mathrm{CH}$ remains dielectric at pressures up to at least $50 \mathrm{GPa}$. The band gap has only a weak dependence on pressure and decreases from 6.67 to $6.42 \mathrm{eV}$ as pressure increases from 0 to $50 \mathrm{GPa}$. For $R_{6}-\mathrm{CH}$, the band gap decreases more rapidly with increasing pressure and reaches $88 \%$ of the original band gap at $50 \mathrm{GPa}$, indicating a stronger pressure dependence similar to those for $\mathrm{K}_{4}-\mathrm{CH}$ and Hex-CH.

We now move on to discuss the optical properties of the $T_{8}$ - $\mathrm{CH}$ and $R_{6}-\mathrm{CH}$ phases in terms of the calculated frequency dependent imaginary part of the dielectric matrix ${ }^{37}$. By comparing the optical spectra at $0 \mathrm{GPa}$ obtained with light polarized either along the $c$ axis or in the $a b$ plane [Fig. 4 (b) and 4(c)], we find that the spectra differ in
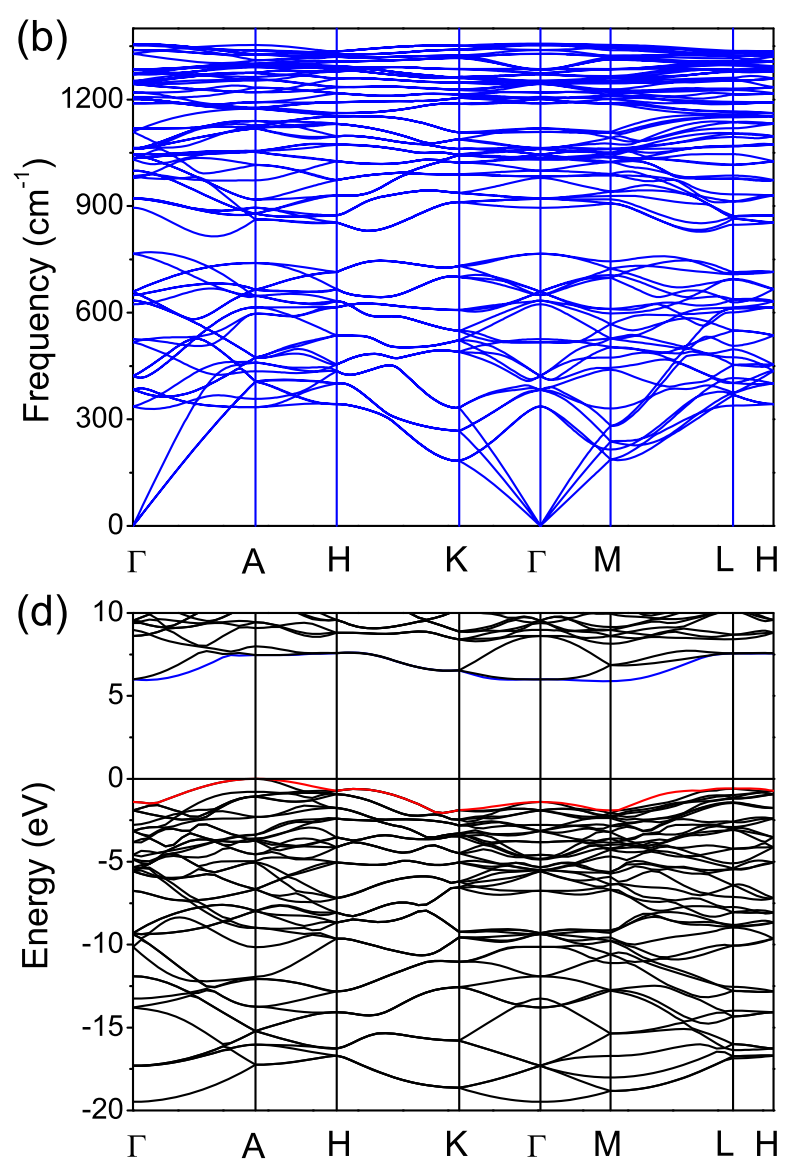

Figure 3 Calculated phonon and electronic band structures at $0 \mathrm{GPa}$. (a) and (b) phonon band structures for $T_{8}-\mathrm{CH}$ and $R_{6}-\mathrm{CH}$. The high frequency C$\mathrm{H}$ stretching modes are given in text. (c) and (d) electronic band structures for $T_{8}-\mathrm{CH}$ and $R_{6}-\mathrm{CH}$. 

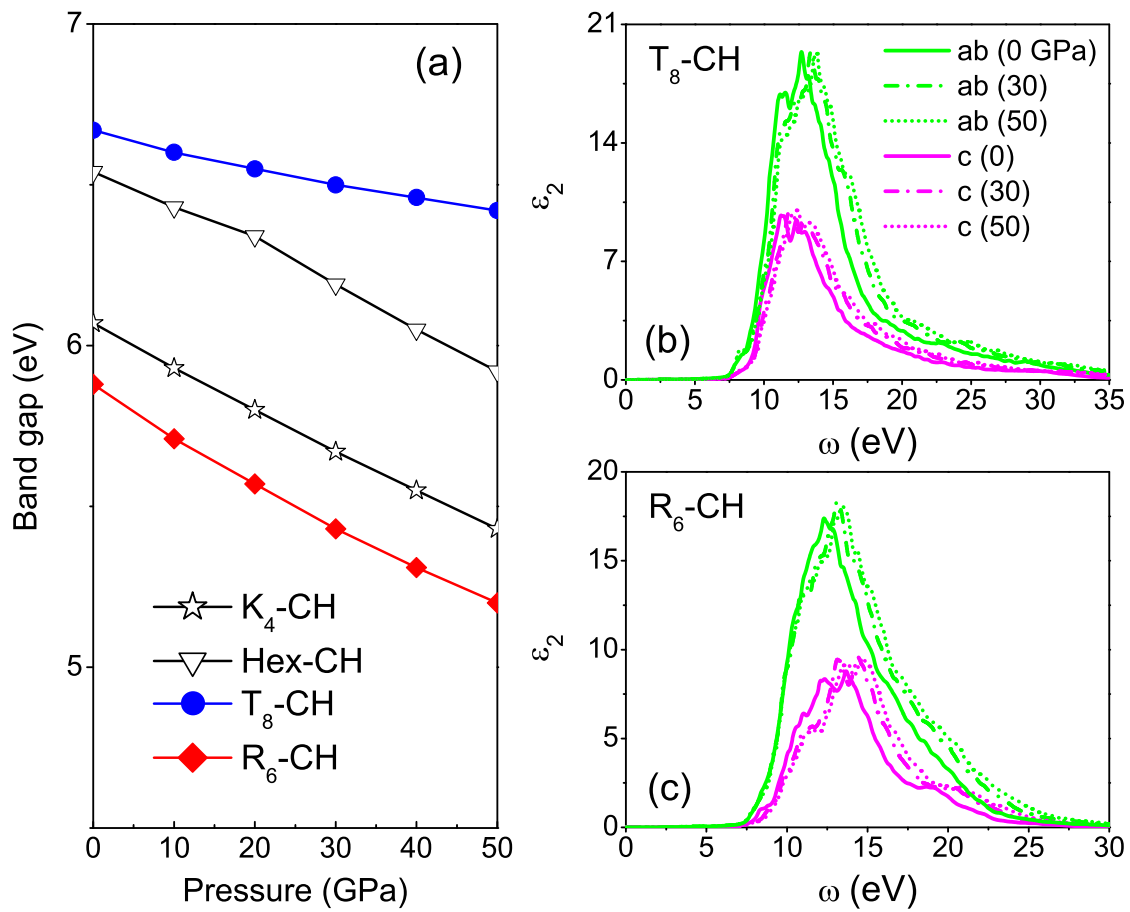

Figure $4 \mid$ (a) Calculated electronic band gaps as a function of pressure for $K_{4}-\mathrm{CH}, \mathrm{Hex}-\mathrm{CH}, T_{8}-\mathrm{CH}$, and $R_{6}-\mathrm{CH}$. (b) and (c) Absorption spectra along the $c$ axis and in the $a b$ plane for $T_{8}-\mathrm{CH}$ and $R_{6}-\mathrm{CH}$ at 0,30 , and $50 \mathrm{GPa}$, respectively.

shape and intensity, suggesting anisotropic features for both chiral phases. The application of pressure on the two structures induces an opposite optical response. By increasing pressure the $\mathrm{C}-\mathrm{C}$ bond length is shortened, the band gap decreases and the whole optical spectrum is almost rigidly shifted toward higher energy. The calculated static dielectric constants for the two chiral phases increase slowly with pressure, with the $a b-(c-)$ components going from 4.045 (4.071) and 3.846 (3.797) at $0 \mathrm{GPa}$ to 4.174 (4.227) and 4.082 (3.934) at $30 \mathrm{GPa}$ for $T_{8}-\mathrm{CH}$ and $R_{6}-\mathrm{CH}$, respectively.

To provide more information and characters for possible experimental observation, we have also simulated the x-ray diffraction (XRD) spectra of the various $\mathrm{CH}$ phases at $0 \mathrm{GPa}$. Monochromatic radiation with a wavelength $1.54056 \AA$ is used, and the results are shown in Fig. 5. Unlike $K_{4}-\mathrm{CH}$ where the main peak (110) at $2 \theta=$ $29.69^{\circ}$ is observed, three sharp XRD peaks of $(101)$ at $25.95^{\circ},(200)$ at $29.23^{\circ}$, and $(211)$ at $39.48^{\circ}$ with strong intensities are seen for $T_{8}-\mathrm{CH}$. Furthermore, we also find significant difference between the two hexagonal phases, with the most prominent peak observed to be (101) at $23.08^{\circ}$ for $\mathrm{Hex}-\mathrm{CH}$ and (110) at $24.06^{\circ}$ for $R_{6}-\mathrm{CH}$, although both phases share the same peak $(20 \overline{1})$ with close positions. Compared with these diamond-like $\mathrm{CH}$ phases, the layered ones have specific XRD peaks at relatively small $2 \theta$ values of $19.70^{\circ}$ for graphane I, $18.46^{\circ}$ for graphane III, and $20.65^{\circ}$ for benzene II polymer, which originate from a large interlayer distance in layered crystal structure. We believe that the above comparison between the chiral hydrocarbons found here and other $\mathrm{CH}$ structures may be helpful for identifying them in experiments.

\section{Discussion}

In summary, we have predicted by ab initio calculations two new 3D hydrocarbon framework structures composed of 3-fold and 4-fold helical $\mathrm{CH}$ chains in $R \overline{3}$ and $I 4_{1} / a$ symmetry, respectively. These saturated crystalline phases are dynamically stable and have large bulk moduli of $\sim 200 \mathrm{GPa}$. We confirm their increasing stabilization with pressure relative to the molecular solids such as acetylene, cubane, and benzene with 1:1 stoichiometry in the pressure range 0-50 GPa. Calculations on the electronic and optical properties

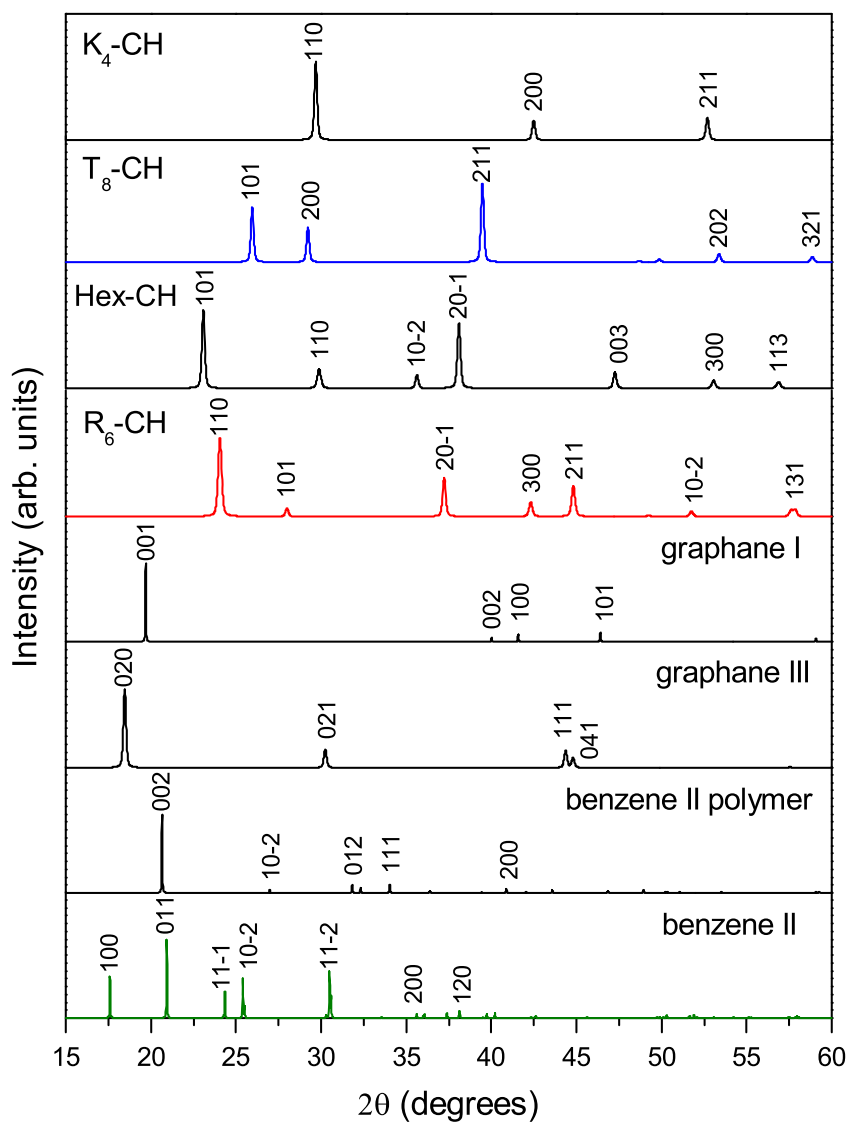

Figure $5 \mid$ Simulated XRD patterns for $K_{4}-\mathrm{CH}, \mathrm{Hex}-\mathrm{CH}, T_{8}-\mathrm{CH}, \mathrm{R}_{6}-\mathrm{CH}$, graphane I and III, benzene II polymer, and benzene II at $0 \mathrm{GPa}$. The Xray wavelength is $1.54056 \AA$. 
show that both chiral hydrocarbons exhibit a wide-gap insulating behavior and anisotropic adsorption spectra. The simulated XRD patterns show the distinct structural feature with respect to the layered $\mathrm{CH}$ phases. The present results will stimulate future experiments on the high-pressure polymerization of molecular hydrocarbons to synthesize the amazing chiral $\mathrm{CH}$ phases, which may have wide applications in mechanical, optoelectronic, and biological materials.

\section{Methods}

The calculations were performed using the density functional theory within the local density approximation (LDA) ${ }^{29,30}$ as implemented in the Vienna ab-initio simulation package (VASP) ${ }^{31}$. We adopted the projector augmented wave (PAW) method ${ }^{32}$ to describe the electron-ion interaction. The plane wave cutoff energy was set to $800 \mathrm{eV}$. Brillouin zone integration was carried out at Monkhorst-Pack ${ }^{33} \mathrm{k}$-point meshes with a grid spacing of $2 \pi \times 0.02 \AA^{-1}$. The geometries were optimized by a conjugate gradient algorithm until the Hellmann-Feynman forces on the ions are less than $10^{-3} \mathrm{eV} / \AA$. Phonon calculations were based on the supercell approach ${ }^{34}$ using the PHONOPY code ${ }^{35}$. The HSE06 hybrid functional method ${ }^{36}$ was employed to calculate the electronic and optical properties. The frequency dependent dielectric matrix was obtained by neglecting local field effects ${ }^{37}$.

1. Mcmillan, P. F. New materials from high-pressure experiments. Nature Mater. 1, 19-25 (2002).

2. Brazhkin, V. V. \& Lyapin, A. G. Metastable high-pressure phases of low-Z compounds: creation of a new chemistry or a prompt for old principles. Nature Mater. 3, 497-500 (2004).

3. Chelazzi, D., Ceppatelli, M., Santoro, M., Bini, R. \& Schettino, V. High pressure synthesis of crystalline polyethylene using optical catalysis. Nature Mater. $\mathbf{3}$, 470-475 (2004).

4. Wieldraaijer, H., Schouten, J. A. \& Trappeniers, N. J. Investigation of the phase diagrams of ethane, ethylene, and methane at high pressures. High Temp. - High Press. 15, 87-92 (1983).

5. Aoki, K. et al. Raman study of the solidstate polymerization of acetylene at high pressure. J. Chem. Phys. 89, 529-534 (1988).

6. Ceppatelli, M., Santoro, M., Bini, R. \& Schettino, V. Fourier transform infrared study of the pressure and laser induced polymerization of solid acetylene. J. Chem. Phys. 113, 5991-6000 (2000).

7. Pruzan, Ph. et al. Transformation of benzene to a polymer after static pressurization to $30 \mathrm{GPa}$. J. Chem. Phys. 92, 6910-6915 (1990).

8. Ciabini, L., Santoro, M., Bini, R. \& Schettino, V. High pressure reactivity of solid benzene probed by infrared spectroscopy. J. Chem. Phys. 116, 2928-2935 (2002).

9. Ciabini, L., Santoro, M., Bini, R. \& Schettino, V. High Pressure Photoinduced Ring Opening of Benzene. Phys. Rev. Lett. 88, 085505 (2002).

10. Cansell, F., Fabre, D. \& Petitet, J. P. Phase transitions and chemical transformations of benzene up to $550^{\circ} \mathrm{C}$ and $30 \mathrm{GPa}$. J. Chem. Phys. 99, 7300-7304 (1993)

11. Eremets, M. I., Gavriliuk, A. G., Trojan, I. A., Dzivenko, D. A. \& Boehler, R. Singlebonded cubic form of nitrogen. Nature Mater. 3, 558-563 (2004).

12. Iota, V., Yoo, C. S. \& Cynn, H. Quartzlike Carbon Dioxide: An Optically Nonlinear Extended Solid at High Pressures and Temperatures. Science 283, 1510-1513 (1999).

13. Somayazulu, M. et al. Novel Broken Symmetry Phase from $\mathrm{N}_{2} \mathrm{O}$ at High Pressures and High Temperatures. Phys. Rev. Lett. 87, 135504 (2001).

14. Sluiter, M. H. F. \& Kawazoe, Y. Cluster expansion method for adsorption: Application to hydrogen chemisorption on graphene. Phys. Rev. B 68, 085410 (2003)

15. Sofo, J. O., Chaudhari, A. S. \& Barber, G. D. Graphane: A two-dimensional hydrocarbon. Phys. Rev. B 75, 153401 (2007).

16. Bhattacharya, A., Bhattacharya, S., Majumder, C. \& Das, G. P. Third conformer of graphane: A first-principles density functional theory study. Phys. Rev. B 83, 033404 (2011).

17. Wen, X. D., Hoffmann, R. \& Ashcroft, N. W. Benzene under High Pressure: a Story of Molecular Crystals Transforming to Saturated Networks, with a Possible Intermediate Metallic Phase. J. Am. Chem. Soc. 133, 9023-9035 (2011).

18. Wen, X. D. et al. Graphane sheets and crystals under pressure. Proc. Natl. Acad. Sci. U.S.A. 108, 6833-6837 (2011).
19. Lian, C. S., Wang, X. Q. \& Wang, J. T. Hydrogenated $\mathrm{K}_{4}$ carbon: A new stable cubic gauche structure of carbon hydride. J. Chem. Phys. 138, 024702 (2013).

20. Itoh, M. et al. New metallic carbon crystal. Phys. Rev. Lett. 102, 055703 (2009).

21. He, C., Sun, L. Z., Zhang, C. X. \& Zhong, J. Low energy three-dimensional hydrocarbon crystal from cold compression of benzene. J. Phys. Condens. Matter 25, 205403 (2013).

22. Wang, J. T., Chen, C. F. \& Kawazoe, Y. New Carbon Allotropes with Helical Chains of Complementary Chirality Connected by Ethene-type $\pi$-Conjugation. Sci. Rep. 3, 3077 (2013).

23. Eaton, P. E. \& Cole, T. W. The Cubane System. J. Am. Chem. Soc. 86, 962-964 (1964).

24. Fleischer, E. B. X-Ray Structure Determination of Cubane. J. Am. Chem. Soc. 86, 3889-3890 (1964).

25. Koski, H. K. \& Sandor, E. Neutron Powder Diffraction Study of the LowTemperature Phase of Solid Acetylene- $\mathrm{d}_{2}$. Acta Crystallogr. B 31, 350-353 (1975).

26. Piermarini, G. J., Mighell, A. D., Weir, C. E. \& Block, S. Crystal Structure of Benzene II at 25 Kilobars. Science 165, 1250-1255 (1969).

27. Murnaghan, F. D. The Compressibility of Media under Extreme Pressures. Proc. Natl. Acad. Sci. U.S.A. 30, 244-247 (1944).

28. Ciabini, L. et al. High-pressure and high-temperature equation of state and phase diagram of solid benzene. Phys. Rev. B 72, 094108 (2005).

29. Ceperley, D. M. \& Alder, B. J. Ground State of the Electron Gas by a Stochastic Method. Phys. Rev. Lett. 45, 566-569 (1980).

30. Perdew, J. P. \& Zunger, A. Self-interaction correction to density-functional approximations for many-electron systems. Phys. Rev. B 23, 5048-5079 (1981).

31. Kresse, G. \& Furthmüller, J. Efficient iterative schemes for ab initio total-energy calculations using a plane-wave basis set. Phys. Rev. B 54, 11169-11186 (1996).

32. Blöchl, P. E. Projector augmented-wave method. Phys. Rev. B 50, 17953-17979 (1994).

33. Monkhorst, H. J. \& Pack, J. D. Special points for Brillouin-zone integrations. Phys. Rev. B 13, 5188-5192 (1976).

34. Parlinski, K., Li, Z.-Q. \& Kawazoe, Y. First-Principles Determination of the Soft Mode in Cubic $\mathrm{ZrO}_{2}$. Phys. Rev. Lett. 78, 4063-4066 (1997).

35. Togo, A., Oba, F. \& Tanaka, I. First-principles calculations of the ferroelastic transition between rutile-type and $\mathrm{CaCl}_{2}$-type $\mathrm{SiO}_{2}$ at high pressures. Phys. Rev. B 78, 134106 (2008).

36. Krukau, A. V., Vydrov, O. A., Izmaylov, A. F. \& Scuseria, G. E. Influence of the exchange screening parameter on the performance of screened hybrid functionals. J. Chem. Phys. 125, 224106 (2006).

37. Gajdos, M., Hummer, K., Kresse, G., Furthmüller, J. \& Bechstedt, F. Linear optical properties in the projector-augmented wave methodology. Phys. Rev. B 73, 045112 (2006).

\section{Acknowledgments}

This study was supported by the National Natural Science Foundation of China (Grant No. 11274356) and the Strategic Priority Research Program of the Chinese Academy of Sciences (Grant No. XDB07000000).

\section{Author contributions}

C.S.L., H.D.L. and J.T.W. designed the study and wrote the paper; C.S.L. carried out ab initio simulations. All authors discussed the results and contributed to the manuscript.

\section{Additional information}

Competing financial interests: The authors declare no competing financial interests.

How to cite this article: Lian, C.-S., Li, H.-D. \& Wang, J.-T. Crystalline structures of polymeric hydrocarbon with 3, 4-fold helical chains. Sci. Rep. 5, 7723; DOI:10.1038/ srep07723 (2015)

This work is licensed under a Creative Commons Attribution 4.0 International License. The images or other third party material in this article are included in the article's Creative Commons license, unless indicated otherwise in the credit line; if the material is not included under the Creative Commons license, users will need to obtain permission from the license holder in order to reproduce the material. To view a copy of this license, visit http://creativecommons.org/licenses/by/4.0/ 